\title{
Central Moment and Multinomial Based Sub Image Clipped Histogram Equalization for Image Enhancement
}

\author{
Kuldip Acharya \\ Department of Computer Science and Engineering, National Institute of Technology, Agartala \\ Barjala, Jirania, Tripura (W), Pin: 799046, India. \\ Email: kuldip.acharjee@gmail.com

\section{Dibyendu Ghoshal} \\ Department of Electronics and Communication Engineering, National Institute of Technology, Agartala \\ Barjala, Jirania, Tripura (W), Pin: 799046, India \\ Email: tukumw@gmail.com
}

Received: 25 March 2020; Accepted: 20 August 2020; Published: 08 February 2021

\begin{abstract}
The visual appearance of a digital image can be improved through image enhancement algorithm by reducing the noise in an image, improving the color, brightness and contrast of an image for more analysis. This paper introduces an image enhancement algorithm. The image histogram is processed through multinomial curvature fitting function to reduces the number of pixels for each intensity value through minimizing the sum of squared residuals. Then resampling is done to smooth out the computed data. After then histogram clipping threshold is computed by central moment processed on the resampled data value to restrict the over enhancement rate. Histogram is equally divided into two sub histograms. The sub histograms are equalized by transfer function to merged the sub images into one output image. The output image is further improved by reducing the environmental haze effect by applying Matlab imreducehaze method, which gives the final output image. Matlab simulation results demonstrate that the proposed method outperforms than other compared methods in terms of both quantitative and qualitative performance evaluation applied on colorfulness based PCQI (C-PCQI), and blind image quality measure of enhanced images (BIQME) image quality metrics.
\end{abstract}

Index Terms: Central Moment, Clipping Threshold, Histogram Equalization, Image Enhancement, Resampling

\section{Introduction}

Histogram equalization is a common technique in spatial domain image enhancement method. Histogram Equalization (HE) is a histogram specification [1] process to equalize the image histogram for image enhancement. Histogram equalization increases the local brightness and contrast of the processed image, to maintained the visual quality of an image while increasing the local contrast [2]. Image enhancement is a significant tool in the branch of digital image processing to produce a higher quality of image through processing the original input image. CLAHE method [3] process on small areas in the image, known as tiles, rather than the total image. Adapthisteq computes the contrast transform method for respective tile separately. Contrast of each individual tile's is enhanced, such that the histogram of the resultant area almost matches the histogram quantified via the 'Distribution' value. The adjacent tiles from a processed image are formerly joint via bilinear interpolation which removes the artificially made limitations. Singh et al. presented exposure-based sub image histogram equalization (ESIHE) [4] for the image shoot in the meager lighting atmosphere. Singh et al. proposed Recursive ESIHE (R-ESIHE) [5] method for low light image enhancement where the histogram is partition by a threshold value computed by an average image intensity value. Median-mean based sub-image clipped histogram equalization (MMSICHE) [6] image enhancement technique is suggested by Singh et al., where an image histogram is separated into four sub histograms by mean and median of histogram. MMSICHE method produces an image which has some artifacts on the resultant image. Brightness preserving dynamic histogram equalization (BPDHE) [7], is an extended version to HE that conserves the mean brightness of the image. This technique smoothed the histogram of the original input image with Gaussian filter and then partitioned the local histogram. Adaptive Gamma Correction with Weighting Distribution (AGCWD) procedure is based on histogram modification system [8] to join histogram equalization process and the gamma correction. Under enhancement and over 
enhancement are two problem of histogram equalization process. The AGCWD process eliminates the disadvantages of both gamma correction and histogram equalization schemes by linking both approaches through a weighting function. In this process gamma correction is convenient for normalized cumulative density function (CDF).

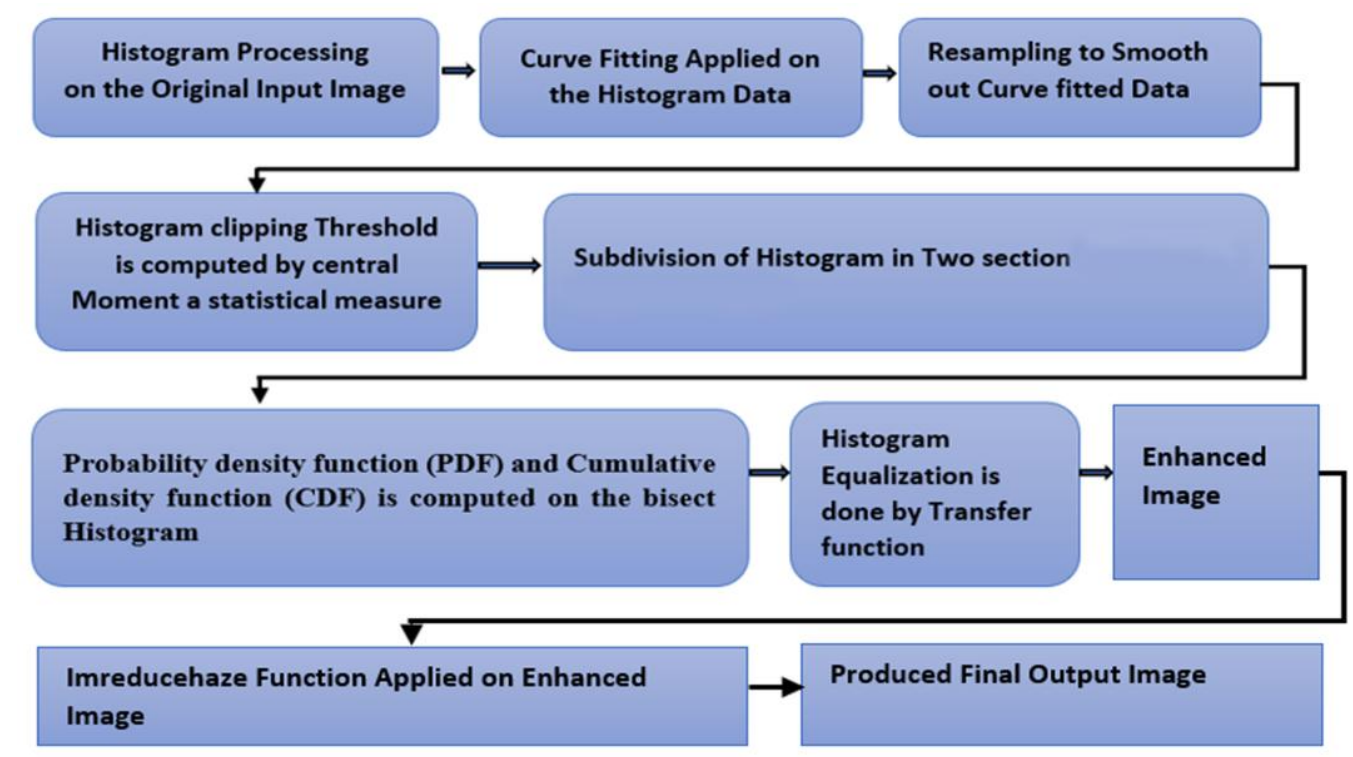

Fig. 1. Flowchart of proposed algorithm

The resultant enhancement image by ESIHE algorithm gives less brightness and contrast compared to MMSICHE, R-ESIHE algorithm. MMSICHE algorithm produced image suffers from artifact. The produced image quality by RESIHE algorithm suffers from over dark effects at some region of output image. AGCWD algorithm gives brighter enhance image but the contrast of produced image is low. CLAHE method resultant image produced less color and contrast compared to ESHIE, MMSICHE and R-ESIHE algorithm. BPDHE method resultant image has some dark effect, less color and contrast. To overcome from these problems the proposed method is introduced to produce superior quality of image with better color and contrast. Among all these image enhancement methods R-ESIHE and BPDHE algorithm provides the best result compared to CLAHE, AGCWD, ESHIE, and MMSICHE. It is observed from the results that the dark region of R-ESIHE algorithm is over darker at some pixels, which degrades the overall performance of image enhancement. BPDHE algorithm resultant image is dull and color quality is poor.

In this paper the major research objective is to produce contrast enhancement images under low light environment. The objective of the proposed algorithm is to produce superior quality of image through contrast enhancement and color improvement.

The remaining paper is arranged in subsequent sections. In section II, literature review is given. The proposed algorithm is offered in section III. The proposed algorithm presented in section IV. Section V presents the simulation results and analysis and section VI gives the conclusion of the paper.

\section{Literature Review}

Traditional histogram equalization (CHE), is a prevalent image enhancement technique, with few limitations. CHE stretches the dynamic range of the image histogram through probability density function (PDF) [9] applied on the image intensity level. CHE highlight high frequency pixels of the histogram and eliminate the low frequency pixels which causes an over enhancement outcome on the output image. Bi-histogram equalization (BBHE) enhancement method is proposed by Chen et al. which mathematically studied to retain the brightness of the original image [10]. Chen et al. proposed minimum mean brightness error bi-histogram equalization (MMBEBHE) algorithm [11] to retain the mean brightness of resultant image. MMBEBHE estimates the absolute mean brightness error (AMBE) of an image histogram and then divide the histogram into two slices grounded on minimum AMBE value. RMSHE technique separated the original image histogram recursively built on the mean of an image. Dualistic sub-image histogram equalization (DSIHE) [12] method is recommended by Yu Wan et al. that retains the brightness and entropy of resultant image. Recursive mean-separate histogram equalization (RMSHE) [13] is an advanced version of Bi-Histogram Equalization (BBHE) [10] method which conserves the image brightness. 


\section{Proposed Algorithm}

- Histogram of color image is computed to find out the intensity value from 0 to 255 and number of pixels for that particular intensity value i.e. how many pixels is there for intensity value 0 to 255 .

-The original histogram value i.e., number of pixels are best fitted by minimizing the sum of squared residuals, which have a minimum square distance from that particular actual intensity value and it is implemented through multinomial curvature fitting method with a specific degree to fit the histogram data.

- The processed data gained from minimum square distance is resampled into a new fixed rate through lowpass filtering method to smooth out the result.

- Histogram is clipped in the proposed method with the threshold value calculated by central moment measure applied on the smooth data obtained from re-sampling process. It is detected from the study of published papers that histogram-based image enhancement methods use a mean or median of the histogram to select a clipping limit. The proposed method offers easiness to calculate the central moment value of image pixel intensity and it is utilized to choose the clipping limit of the image histogram.

-Histogram is equally partitioned into two sections by using sub histogram limit parameter $\mathrm{X}_{\mathrm{p}}$

-After then histogram equalization is accomplished through transfer functions and then integrated it into a single output image.

-The output enhance image is further processed to reduce the environmental haze effect using imreducehaze [14, 15] function of Matlab which produces the final output image.

-Performance evaluation is done by Matlab [16] on the proposed algorithm over 100 images which are the publicly available in BSD dataset [17]. It is found from the relative study that the proposed method is superior to many other prevailing methods in terms of colorfulness based PCQI (C-PCQI) [18] and blind image quality measure of enhanced images (BIQME) [18]. The present algorithm demonstrates an image enhancement method which keeps both the contrast and color quality of images at an optimal level.

Flowchart of the proposed algorithm is illustrated in Fig.1. Here histogram of an image is fitted by statistical method through minimizing the sum of squared residuals to reduce the actual distance through multinomial curvature fitting technique followed by resampling of fitted data. Then clipping threshold is calculated by central moment on the resampled data to restrict the ratio of over enhancement. Then histogram is equally partition into two part followed by equalization of sub image histogram. Histogram sub division process is shown in Fig.2. Maximum image intensity is L which is 256 .

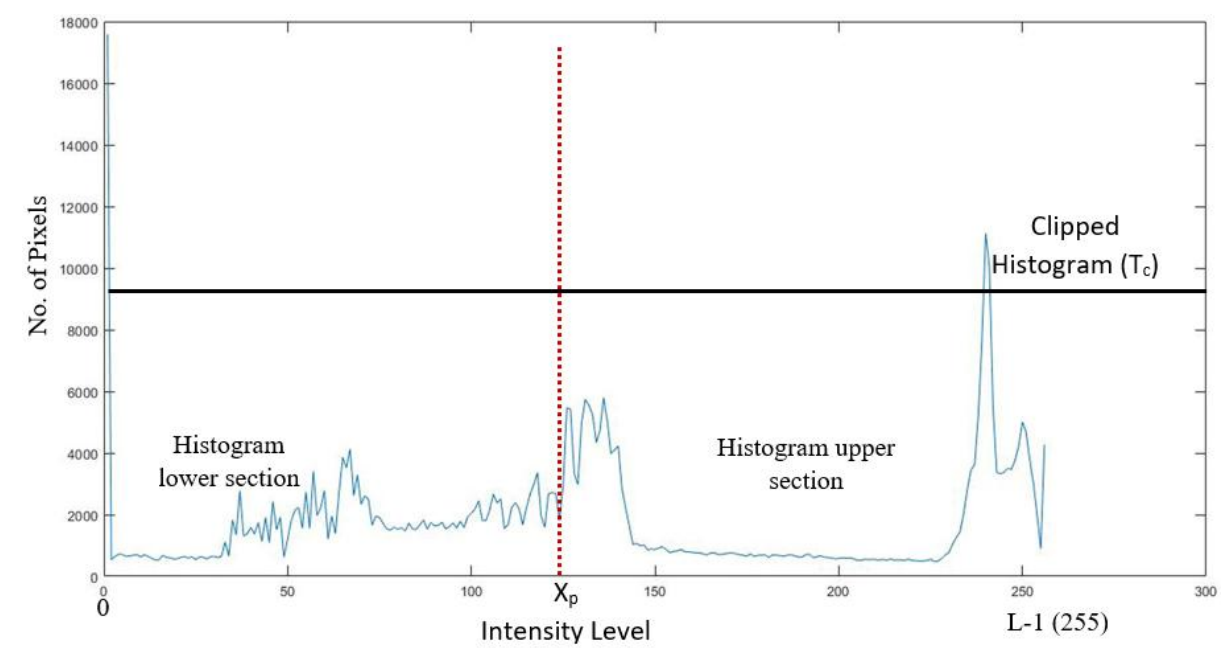

Fig. 2. Histogram sub division process

Then 'imreducehaze' function is applied to reduce the environmental haze effect and the final output enhance image is produced.

\section{Proposed Methodology}

\section{A. Image pixel calculation}

Total number of image pixel $\left(\mathrm{T}_{\mathrm{P}}\right)$ is computed in equation (1) as 


$$
T_{P}=\sum_{i p=0}^{L-1} H(i p)
$$

Here, H(ip) is the histogram of original image and L denotes the maximum image intensity level which is range from 0 to 255 .

Mean value $(\mu)$ computation is specified in equation (2) as:

$$
\mu=\frac{\sum_{i p=0}^{L-1} i p \times H(i p)}{\sum_{i p=0}^{L-1} H(i p)}
$$

\section{B. Multinomial curvature fitting process}

Multinomial curvature fitting [19,20] fit the data of pixels intensity value by minimizing the sum of squared residuals to reduce the distance of original data from that particular actual value. Multinomial curvature fitting with degree 6 try to best fit the data is expressed in (3) as:

$$
\mathrm{P}_{\mathrm{CF}}=\mathrm{aP}^{6}+\mathrm{bP}^{5}+\mathrm{cP}^{4}+\mathrm{dP}^{3}+\mathrm{eP}^{2}+\mathrm{fP}+\mathrm{g}
$$

$\mathrm{P}_{\mathrm{CF}}$ is a multinomial of degree 6. Here $6,5,4,3$, and 2 are multinomial degree.

\section{Resample}

Matlab 'resample' [21] is a method which linearly do a classification of original pixels at a given rate in Hertz (Hz). Plot of an original and resampled intensity pixel of an image is shown in Fig. 3. The original data is represented by 'blue stars' and resample data is represented by 'red circles.

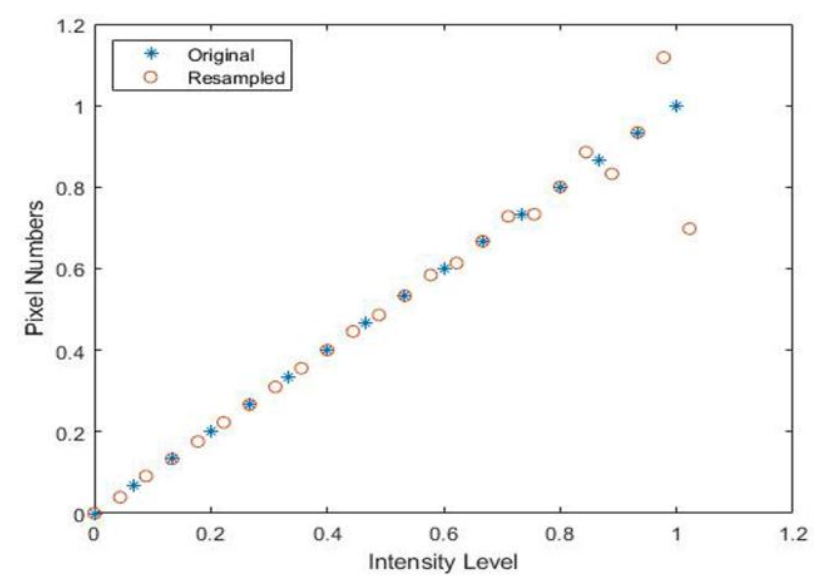

Fig.3 Original and Resample Pixel Values

Matlab Syntax of Resample:

$\mathrm{RS}=$ resample $(\mathrm{u}, \mathrm{v}, \mathrm{w})$ resamples the original input pixels in order, $\mathrm{u}$, at $\mathrm{v} / \mathrm{w}$ times the original pixel proportion. Here Matlab 'resample' [21] function do resample of data to a predefined sampling rate.

\section{Clipping Threshold calculation}

The central moment of order $\mathrm{k}$ for a distribution where $\mu$ is the mean of $x$, and $E V(t)$ represents the expected value of the quantity $t$. The moment function computes a sample version of this pixel intensity value.

$$
M_{i p}=E V(\mathrm{RS}-\mu)^{i p}
$$

$\mathrm{M}_{\mathrm{ip}}$ is a calculated central moment value. RS is computed resample value.

Clipped histogram threshold $\left(\mathrm{T}_{\mathrm{H}}\right)$ value computation using central moment is expressed in equation (7) 


$$
T_{H}=\frac{1}{L} \sum_{i p=1}^{L}\left(R S_{i p}-\overline{R S}\right)^{i p}
$$

Histogram Clipping is represented in equation (8)

$$
H_{C}(\mathrm{ip})= \begin{cases}T_{H} & \text { if } H(\mathrm{ip}) \geq \mathrm{T}_{H} \\ G(\mathrm{ip}) \text { if } \mathrm{H}(\mathrm{ip})<T_{H}\end{cases}
$$

E. Median based Histogram Subdivision

$$
M=\frac{G(\mathrm{ip})}{2}
$$

F. Probability density function $(P D F)$

$\mathrm{PD}_{\mathrm{lw}}$ (i) and $\mathrm{PD}_{\mathrm{up}}$ (i) are equivalent PDF of the sub images as follows:

$$
\begin{gathered}
\mathrm{PD}_{\mathrm{lw}}(\mathrm{i})=\mathrm{H}_{\mathrm{c}}(\mathrm{ip}) / \mathrm{SI}_{\mathrm{i}} \text { for } 0 \leq \mathrm{ip} \leq \mathrm{X}_{\mathrm{m}} \\
\mathrm{PD}_{\mathrm{up}}(\mathrm{i})=\mathrm{H}_{\mathrm{c}}(\mathrm{ip}) / \mathrm{SI}_{\mathrm{u}} \text { for } \mathrm{X}_{\mathrm{m}+\mathrm{i}} \leq \mathrm{ip} \leq \mathrm{L}-1
\end{gathered}
$$

Where $\mathrm{SI}_{\mathrm{i}}$ and $\mathrm{SI}_{\mathrm{u}}$ are a total number of pixels in sub-images $\mathrm{PD}_{\mathrm{lw}}$ and $\mathrm{PD}_{\mathrm{up}}$ respectively.

G. Cumulative density function $(C D F)$

CDF of distinct sub-images expressed as

$$
\begin{gathered}
C D_{l w}(i p)=\sum_{i p=0}^{X_{i p}} P D_{l w}(\mathrm{ip}) \\
C D_{u p}(i p)=\sum_{i p=X_{m+1}}^{L-1} P D_{u p}(\mathrm{ip})
\end{gathered}
$$

The next step of the proposed algorithm is to equalize both the sub histograms individually. The transfer function for histogram equalization based on equation (14) and (15) can be defined as

$$
\begin{gathered}
\mathrm{TR}_{\mathrm{l}}=\mathrm{X}_{\mathrm{m}} \times \mathrm{CD}_{\mathrm{lw}} \\
\mathrm{TR}_{\mathrm{U}}=\left(\mathrm{X}_{\mathrm{m}}+1\right)+\left(\mathrm{L}-\mathrm{X}_{\mathrm{m}}+1\right) \mathrm{CD}_{\mathrm{up}}
\end{gathered}
$$

$\mathrm{TR}_{1}$ and $\mathrm{TR}_{\mathrm{U}}$ are transfer function utilized for equalizing the sub histograms individually. The next step is to merge both sub-images into one final image. The enhance output image is produced by a combination of both transfer functions.

The resultant image is more enhance by dropping the haze effect causes through environmental condition via Matlab imreducehaze function, which produces the final output image.

\section{Simulation Results and Analysis}

\section{A. Quantitative Evaluation and Simulation Results}

Performance assessment is accomplished by Matlab [16] simulation on to quantify visual quality of proposed and other compared methods in terms of colorfulness based PCQI (C-PCQI) [18], and blind image quality measure of enhanced images (BIQME) [18] image quality metric over the BSD image dataset [17]. Machine configuration is Intel Core $\mathrm{i} 7$ processor, 8 G.B. Random access memories on Windows operating system.

B. The Colorfulness Based PCQI (C-PCQI) [18] 
The PCQI image quality metric is correlate through subjective quality scores [16] used on images, that never reflect the influence of colorfulness, which is a dynamic aspect of image quality calculation. The colorfulness based PCQI (C-PCQI) metric solve this problem. The Colorfulness based PCQI (C-PCQI) metric is stated as

$$
S_{C P C Q I}=\frac{1}{N} \sum S I_{m k}(k) \cdot \mathrm{SI}_{c c}(k) \cdot \mathrm{SI}_{s d}(k) \cdot \mathrm{SI}_{c s}(k)
$$

$\mathrm{N}$ is the number of pixels. Here, $\mathrm{SI}_{\mathrm{mk}}$, indicate the similarity over mean intensity among the input image and distorted image, $\mathrm{SI}_{\mathrm{cc}}$ imply the contrast variation and $\mathrm{SI}_{\mathrm{sd}}$ signify the structural distortion among the input image and distorted image. Information about these three positions are specified in [18].

$$
S I_{C S}=\left(\frac{2 O T_{1} \cdot O T_{2}+\square}{O T_{1}^{2} \cdot O T_{2}^{2}+\square}\right) \Omega
$$

The color saturation of the original and distorted images is denoted by $\mathrm{OT}_{1}$ and $\mathrm{OT}_{2}$, respectively. A very small constant number $\square$ is applied to restricts division-by-zero and a fixed pooling index signified by $\Omega$ applied for emphasizing the position which have exceptional variations of image color saturation. Table 2 gives the C-PCQI outcomes of various methods. It is observed that the present method generates the highest C-PCQI values for all the processed BSD images which is 1.1277. The second-best C-PCQI value is given by BPDHE method which is 1.0921 .

\section{Blind image quality measure of enhanced images (BIQME) [18]}

BOIEM metric, an enhanced method of RICE and AGCWD methods are used by three constraints $\partial_{b}, \partial_{\mathrm{s}}, \partial_{\mathrm{c}}$ followed by BIQME method that optimize these three constraints:

$$
\partial_{b}, \partial_{\mathrm{s},} \partial_{c}=\operatorname{maximize}_{\partial_{b}, \partial_{\mathrm{s}}, \partial_{\mathrm{c}}} J_{A}\left(\mathrm{~K}_{B}\left[\mathrm{~K}_{C}\left(s, \partial_{b}\right), \partial_{\mathrm{s},} \partial_{\mathrm{c}}\right]\right)
$$

Where $\mathrm{J}_{\mathrm{A}}, \mathrm{K}_{\mathrm{B}}$ and $\mathrm{K}_{\mathrm{C}}$ are related to BIQME. After then, these parameters are used to image enhancement.

Table 1, demonstrates the BIQME results of numerous compared methods. It is observed from the simulation results that the present method generates the highest BIQME values for all the processed BSD images which is 0.68034 . A higher BIQME [18] specifies improved image quality. The second-best BIQME value is given by BPDHE method which is 0.64050 .

Table 1. Blind image quality measure of enhanced images (BIQME)

\begin{tabular}{cccccccc}
\hline BSD File & RESIHE & ESIHE & AGCWD & BPDHE & CLAHE & MMSICHE & Proposed \\
\hline "106047" & 0.64004 & 0.63706 & 0.20270 & 0.63881 & 0.56392 & 0.59981 & 0.69545 \\
"293029" & 0.66689 & 0.66263 & 0.32454 & 0.65217 & 0.63195 & 0.66613 & 0.70049 \\
"296059" & 0.63824 & 0.63068 & 0.21984 & 0.61661 & 0.59285 & 0.62529 & 0.66110 \\
"317043" & 0.63594 & 0.62224 & 0.28474 & 0.67980 & 0.57089 & 0.63039 & 0.71575 \\
"346016" & 0.62274 & 0.60740 & 0.20082 & 0.64139 & 0.58761 & 0.64865 & 0.70001 \\
"6046" & 0.64995 & 0.64440 & 0.20164 & 0.65223 & 0.62689 & 0.63275 & 0.65484 \\
"80085" & 0.61163 & 0.60774 & 0.28942 & 0.60249 & 0.59600 & 0.59042 & 0.63480 \\
Average & $\mathbf{0 . 6 3 7 9 1 9}$ & $\mathbf{0 . 6 3 0 3 0}$ & $\mathbf{0 . 2 4 6 2 4}$ & $\mathbf{0 . 6 4 0 5 0}$ & $\mathbf{0 . 5 9 5 7 3}$ & $\mathbf{0 . 6 2 7 6 3}$ & $\mathbf{0 . 6 8 0 3 4}$ \\
\hline
\end{tabular}

Table 2. Colorfulness based PCQI (C-PCQI)

\begin{tabular}{clllllll}
\hline BSD File & RESIHE & ESIHE & AGCWD & BPDHE & CLAHE & MMSICHE & Proposed \\
\hline "106047" & 1.1095 & 1.0720 & 0.11761 & 1.1101 & 1.0258 & 1.0477 & 1.1451 \\
"293029" & 1.0805 & 1.0665 & 0.04189 & 1.0942 & 1.0326 & 1.0586 & 1.1423 \\
"296059" & 1.0607 & 1.0380 & 0.13530 & 1.0432 & 1.0221 & 1.0347 & 1.0856 \\
"317043" & 1.1254 & 1.0807 & 0.02535 & 1.2270 & 1.0294 & 1.1329 & 1.2454 \\
"346016" & 1.0606 & 1.0337 & 0.01350 & 1.1345 & 1.0276 & 1.0900 & 1.1929 \\
"6046" & 1.0545 & 1.0456 & 0.06221 & 1.0340 & 1.0253 & 1.0043 & 1.0577 \\
"80085" & 1.0126 & 1.0055 & 0.34123 & 1.0018 & 1.0043 & 0.9715 & 1.0250 \\
Average & $\mathbf{1 . 0 7 1 9}$ & $\mathbf{1 . 0 4 8 8}$ & $\mathbf{0 . 1 0 5 3 0}$ & $\mathbf{1 . 0 9 2 1}$ & $\mathbf{1 . 0 2 3 8}$ & $\mathbf{1 . 0 4 8 5}$ & $\mathbf{1 . 1 2 7 7}$ \\
\hline
\end{tabular}


Fig. 4(a) illustrates the original BSD image '296059' of 'Elephant' and the result of present method in Fig 4(b). Fig. 4(c) shows the histogram of original image and Fig. 4(d) illustrates the histogram of proposed method. It is clear from the result that the output image is much improved than original image and showing superior quality of image as an image enhancement result.

The tonal distribution of a digital image is represented by a graphical illustration [22] known as image histogram. The left portion of the horizontal axis of histogram signifies the dark areas, the middle portion of histogram signifies mid-tone values and the right-hand portion of histogram signifies light zones. The vertical axis shows the size of the total number of pixels which is occupied individually one of these regions. Histogram of the proposed and different compared methods is shown in Fig.5-9. It's clearly observed from the histogram specification that the pixels of the proposed method histogram keep an optimum balance in the left, middle and right zones of histogram and proves its excellence than other compared methods.

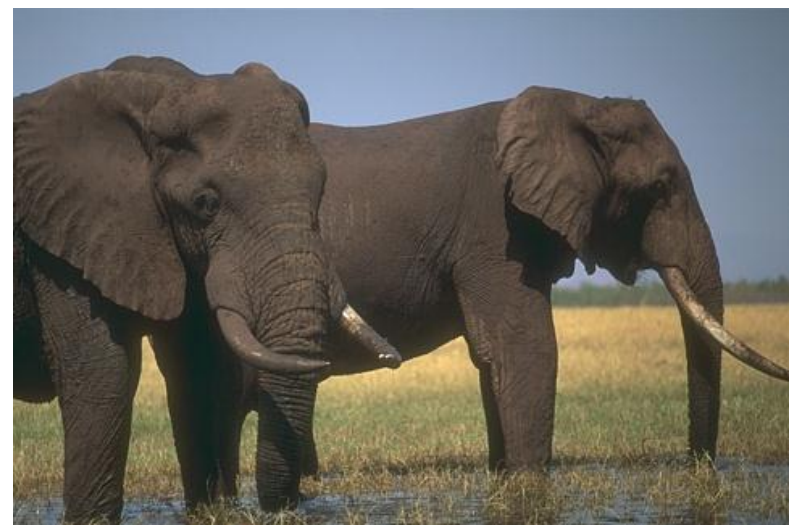

(a) Original BSD Image ‘296059’ of Elephants

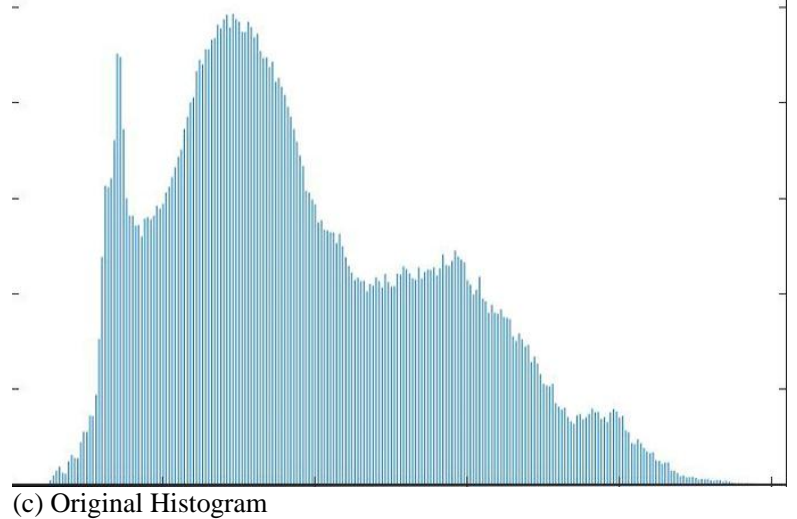

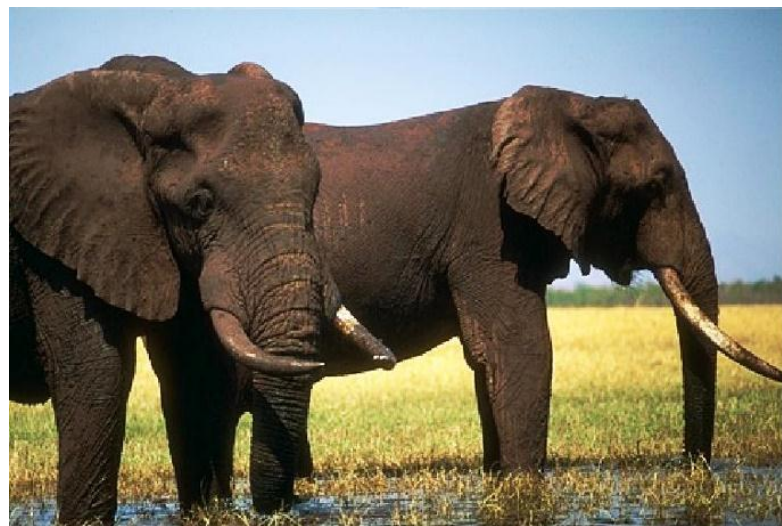

(b) Proposed Method

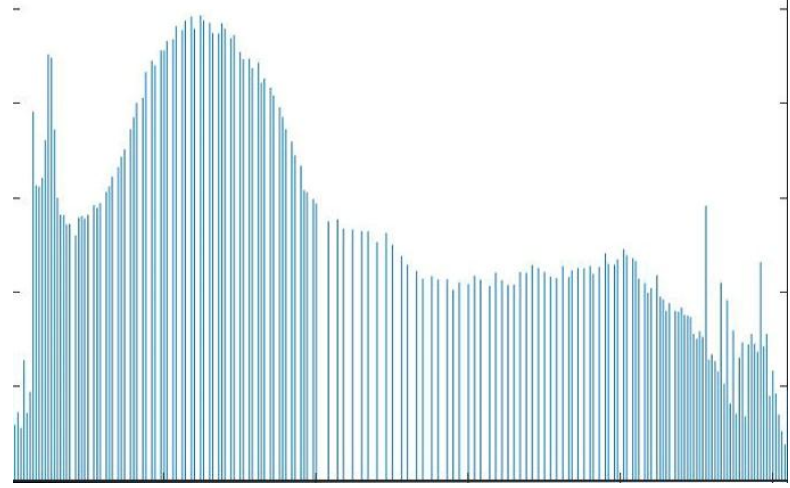

(d) Histogram of Proposed Method

Fig. 4 (a) Original image (b) proposed method (c) Original image histogram (d) Proposed method image histogram

Fig. 5 demonstrates the results of present algorithm and other compared enhancement algorithm processed on the original BSD image ' 80085 ' of 'Flying cloth'. The image enhancement results given by ESIHE algorithm show a low light image with less brightness and contrast. MMSICHE algorithm demonstrates a slightly improved image than ESIHE algorithm, but it has less contrast image. R-ESIHE algorithm produced an improved contrast enhance image than ESIHE and MMSICHE algorithm, but have a dark effect at some area. AGCWD algorithm produces a brighter image with low contrast. CLAHE method illustrates an image with less color and contrast compare to ESHIE, MMSICHE and R-ESIHE algorithm. BPDHE method illustrates an image close to AGCWD but have some dark effect, less color and contrast compare to ESHIE, MMSICHE and R-ESIHE algorithm. The overall visual quality of the proposed algorithm is better than the compared algorithms. The proposed algorithm demonstrates a vibrant image which keeps the color quality with finest contrast and brightness.

Fig. 6 reveals the outcomes of present method and other related enhancement method processed on the original BSD image '106047' of 'Penguin'. The enhancement outcomes offered by ESIHE algorithm shows a low light image with less brightness and contrast. MMSICHE algorithm illustrates a little contrast enhanced image than ESIHE method. R-ESIHE method formed a contrast enhanced image than ESIHE and MMSICHE method. Some area of an output image, by this method, has a shadowy effect. AGCWD method generates a brighter image with little contrast. CLAHE method demonstrates an image with a smaller amount of color and contrast in comparison to ESHIE, MMSICHE and R-ESIHE methods. BPDHE method demonstrates an image almost similar to AGCWD but has some dim effect, a 
smaller amount color and contrast compare to ESHIE, MMSICHE and R-ESIHE. The overall photographic superiority of the proposed method is improved than the other related methods. The proposed method illustrates a vibrant image that preserves the color quality with best contrast, and brightness.

Fig. 7 shows the results of present algorithm and other compared enhancement algorithm processed on the original BSD image '293029' of 'Seller'. ESIHE displays a low light, less brightness and contrast image. MMSICHE shows a slightly improved image than ESIHE, but it has a smaller amount contrast. R-ESIHE algorithm formed a contrast enhance image compare to ESIHE and MMSICHE methods with few dark effects at some location of output image. AGCWD gives a low contrast image than other compared methods. CLAHE method shows a less contrast color image in comparison to ESHIE, MMSICHE and R-ESIHE method. BPDHE method demonstrates an image close to AGCWD with few dark pixels, less contrast, and color in comparison to ESHIE, MMSICHE and R-ESIHE algorithm. The overall pictorial superiority of the present method is improved than the other associated methods. The proposed method shows a vibrant image that retains the color quality with optimum contrast, and brightness.

Fig. 8 demonstrates the results of present algorithm and other compared enhancement algorithm processed on the original BSD image '317043' of 'Old Garden'. ESIHE method shows a low brightness and contrast image. MMSICHE method shows a less contrast enhanced image than ESIHE method. R-ESIHE method formed a contrast enhance image than ESIHE and MMSICHE method, with a shady effect at some part of output image. AGCWD shows a brighter image but have a low contrast. CLAHE demonstrates an image with a smaller amount color and contrast in comparison to ESHIE, MMSICHE and R-ESIHE method. BPDHE method illustrates an image close to AGCWD but have some dark effect, less color and contrast compare to ESHIE, MMSICHE and R-ESIHE algorithm. The overall photographic eminence of the proposed method is improved than the other associated compared methods. The proposed method shows a vibrant image that preserves the color superiority with optimum contrast, and brightness.

Fig. 9 illustrates the results of present methods and other related compared methods processed on the original BSD image '346016' of 'Deer'. ESIHE displays a less brightness and contrast image. MMSICHE illustrates a little enhanced image than ESIHE, with less contrast image. R-ESIHE displays an improved contrast enhance image than ESIHE and MMSICHE with few dark effects at some part of output image. AGCWD displays a bright image but with low contrast effect. CLAHE demonstrates an image with fewer colors and contrast relate to ESHIE, MMSICHE and R-ESIHE. BPDHE method demonstrates an image close to AGCWD but has some dark effect, less color and contrast compare to ESHIE, MMSICHE and R-ESIHE. The proposed method illustrates a vibrant image which preserves the color quality with optimum contrast, and brightness.

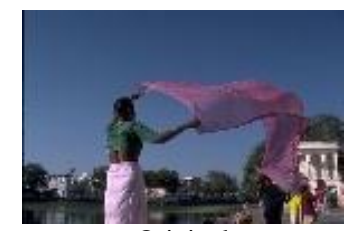

Original

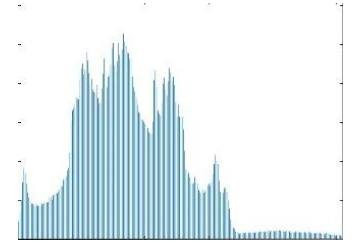

Original Histogram

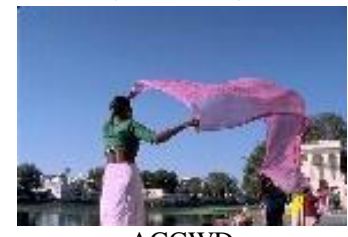

AGCWD

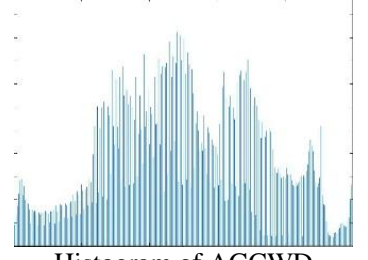

Histogram of AGCWD

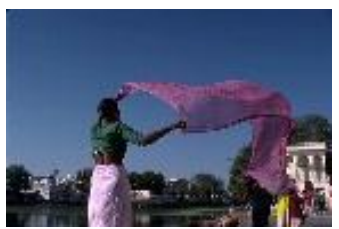

ESIHE

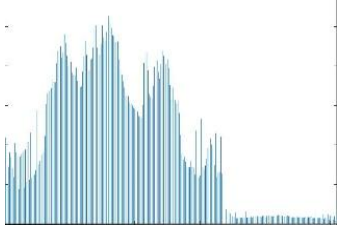

Histogram of ESIHE

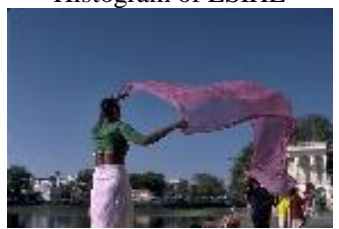

CLAHE

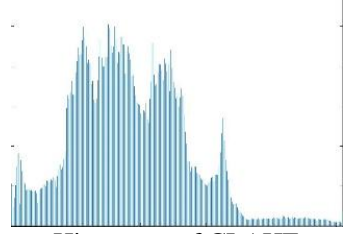

Histogram of CLAHE

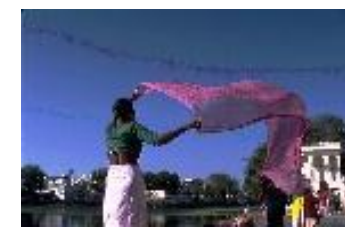

MMSICHE
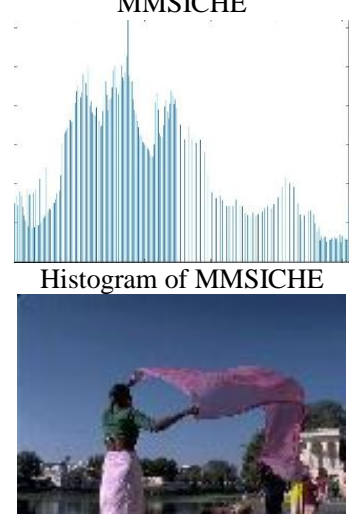

BPDHE

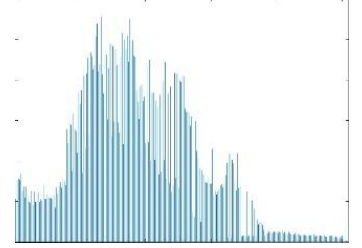

Histogram of BPDHE

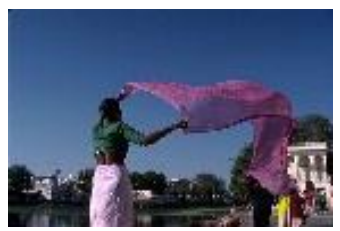

R-ESIHE

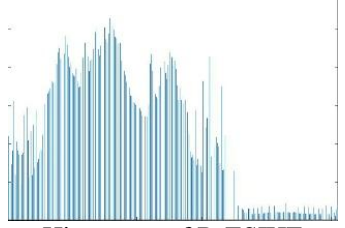

Histogram of R-ESIHE

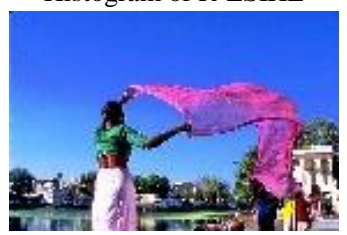

Proposed

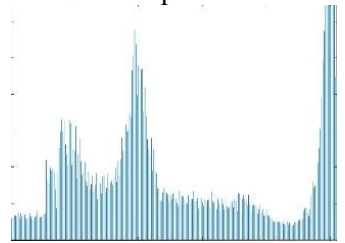

Histogram of Proposed

Fig. 5. BSD Image 80085of 'Flying cloth' and output of different image enhancement methods 


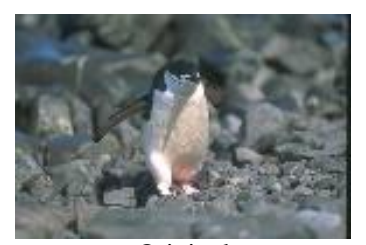

Original

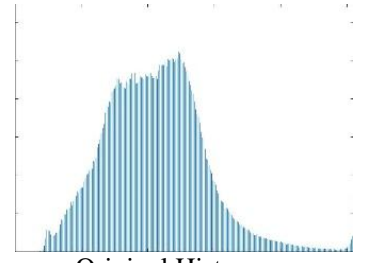

Original Histogram

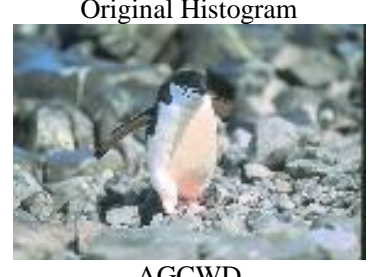

AGCWD

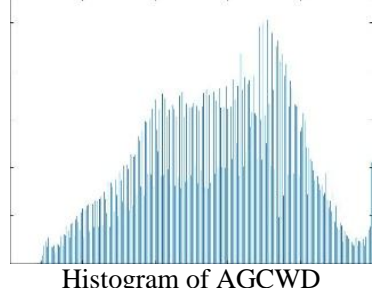

Histogram of AGCWD

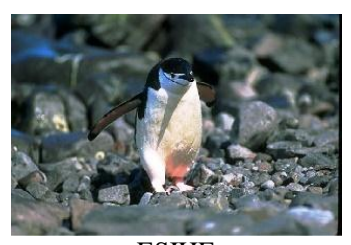

ESIHE

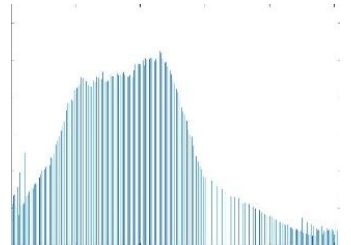

Histogram of ESIHE

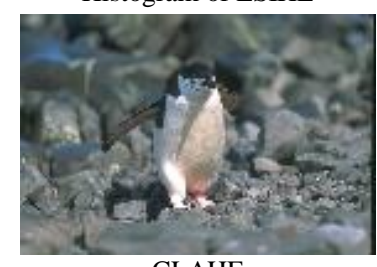

CLAHE

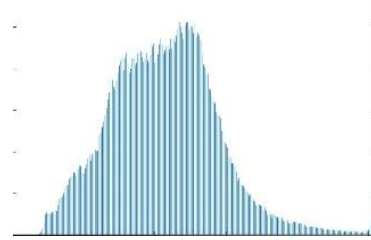

Histogram of CLAHE

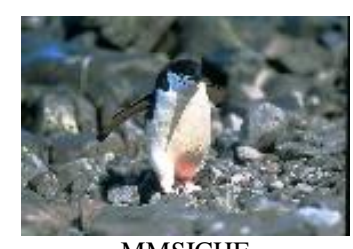

MMSICHE

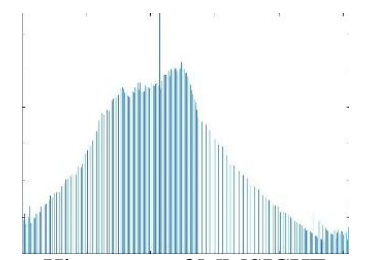

Histogram of MMSICHE

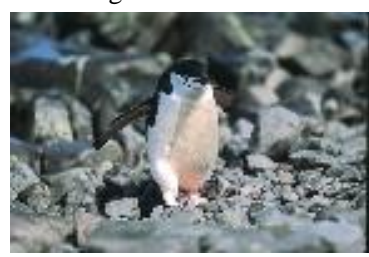

BPDHE

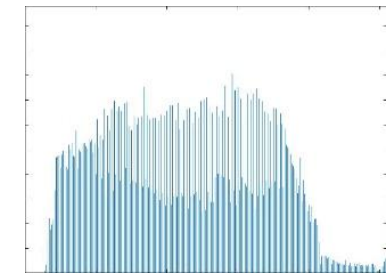

Histogram of BPDHE

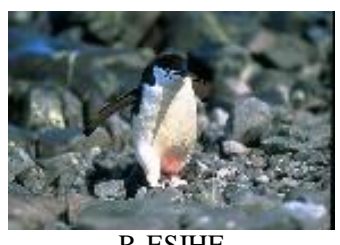

R-ESIHE

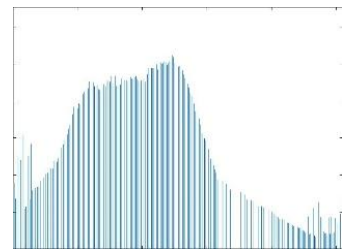

Histogram of R-ESIHE

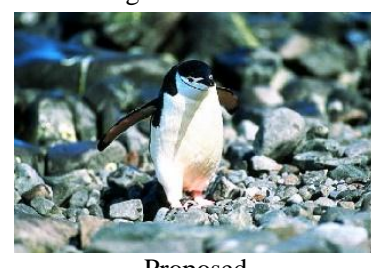

Proposed

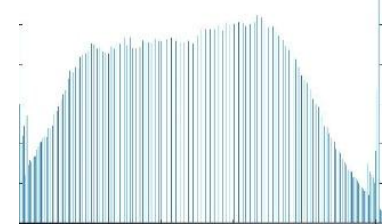

Histogram of Proposed

Fig. 6. BSD Image '106047' of 'Penguin' and output of different image enhancement methods

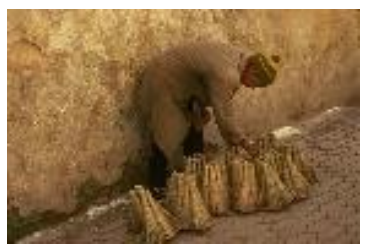

Original

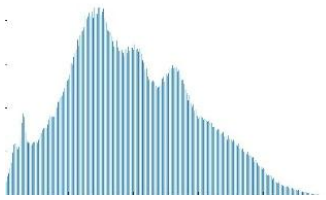

Original Histogram

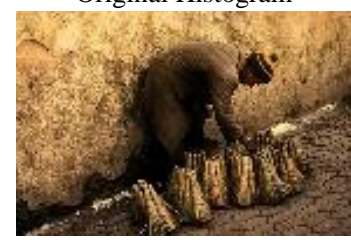

AGCWD

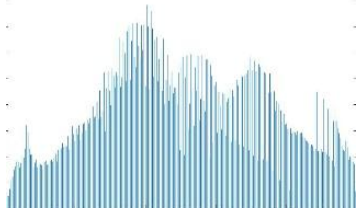

Histogram of AGCWD

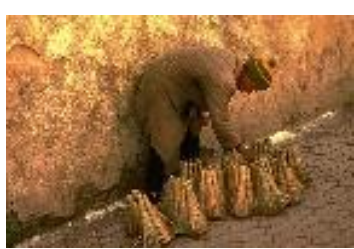

ESIHE

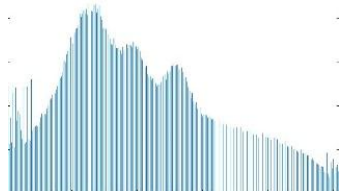

Histogram of ESIHE

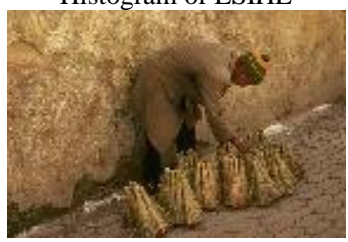

CLAHE

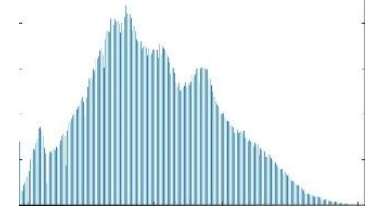

Histogram of CLAHE

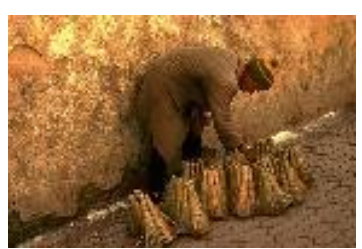

MMSICHE

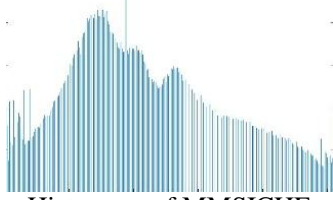

Histogram of MMSICHE

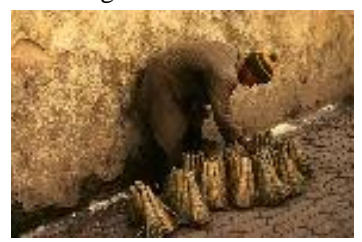

BPDHE

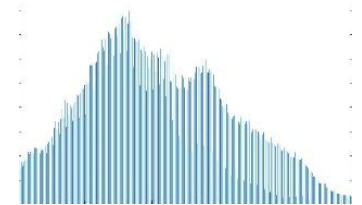

Histogram of BPDHE

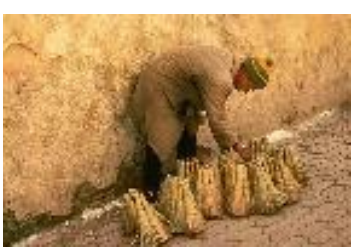

R-ESIHE

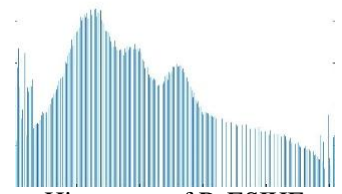

Histogram of R-ESIHE

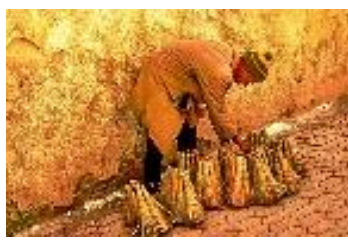

Proposed

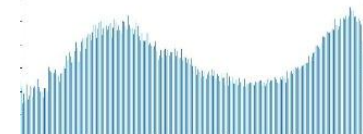

Histogram of Proposed

Fig. 7. BSD Image 293029 of 'Seller' and output of different image enhancement methods 


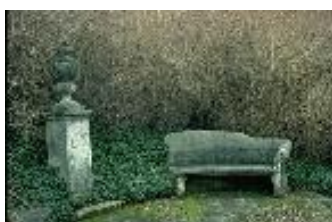

Original

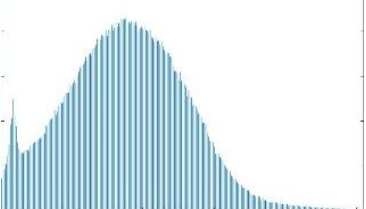

Original Histogram

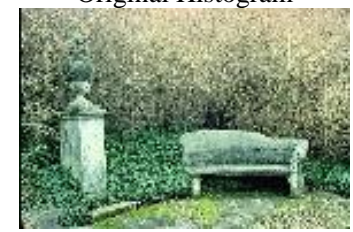

AGCWD

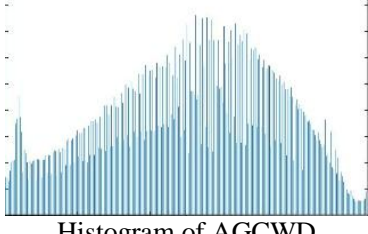

Histogram of AGCWD

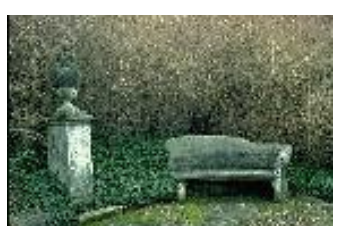

ESIHE

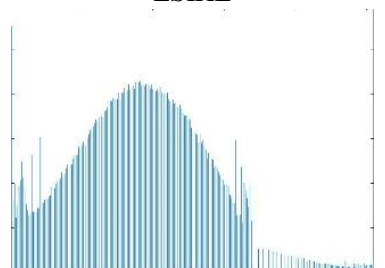

Histogram of ESIHE

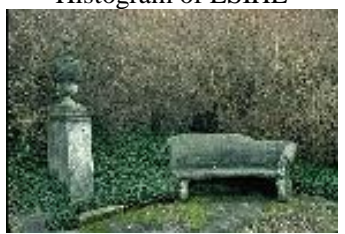

CLAHE

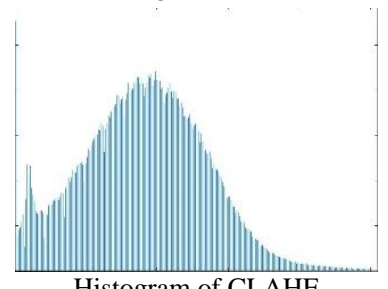

Histogram of CLAHE

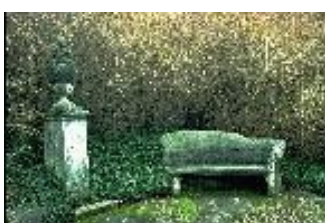

MMSICHE

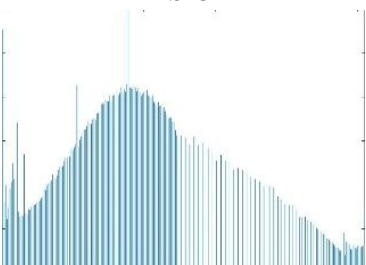

Histogram of MMSICHE

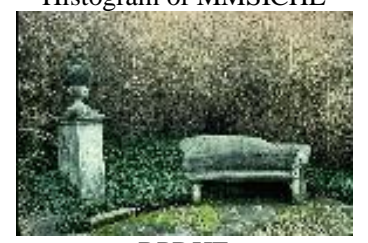

BPDHE

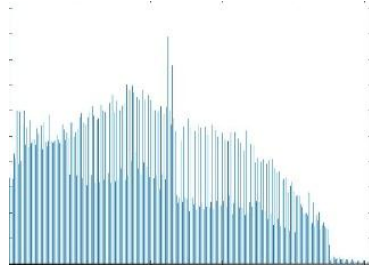

Histogram of BPDHE

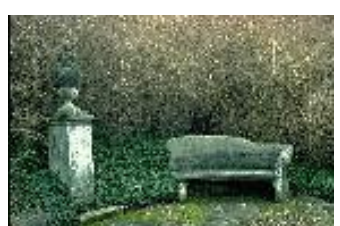

R-ESIHE

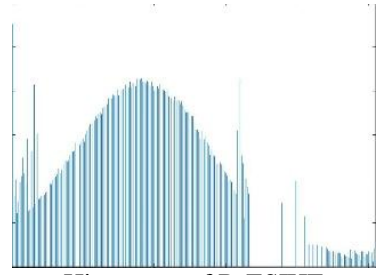

Histogram of R-ESIHE

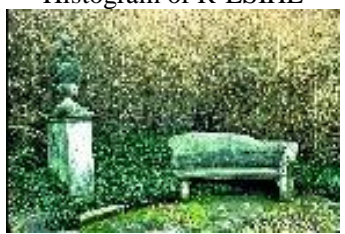

Proposed

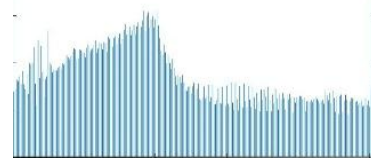

Histogram of Proposed

Fig.8. BSD Image '317043' of 'Old Garden' and output of different image enhancement methods

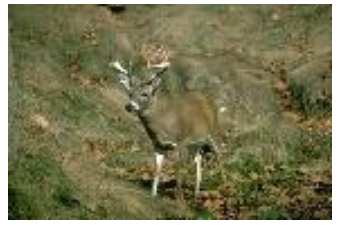

Original

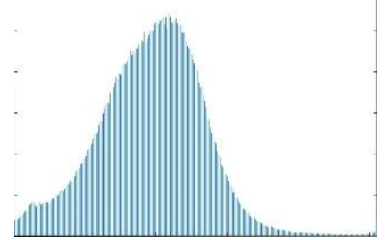

Original Histogram

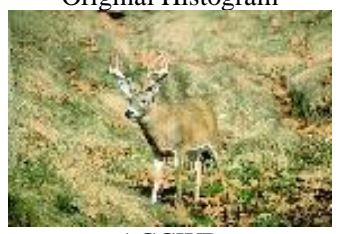

AGCWD

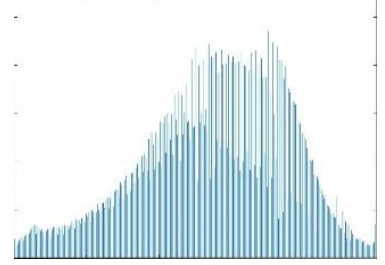

Histogram of AGCWD

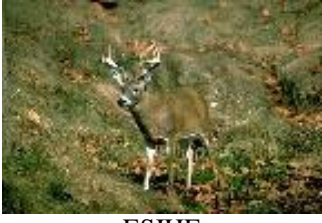

ESIHE
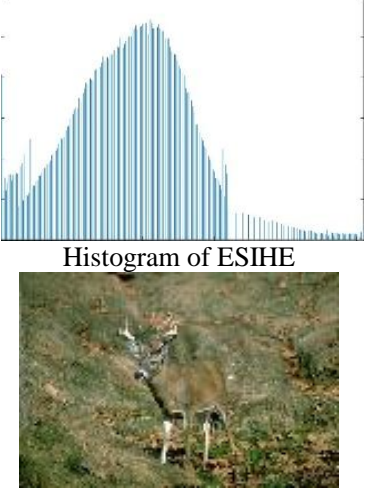

CLAHE

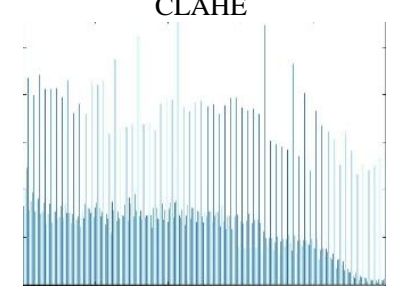

Histogram of CLAHE

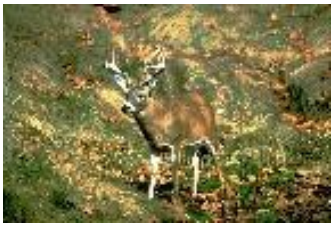

MMSICHE
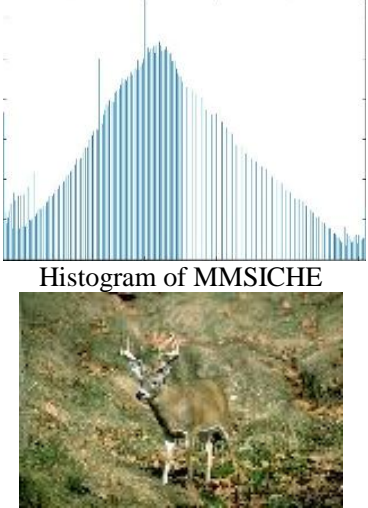

BPDHE

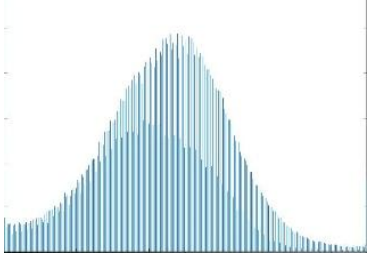

Histogram of BPDHE

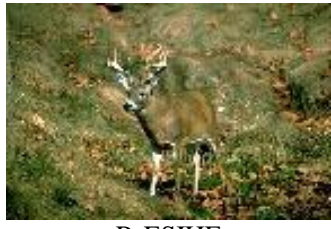

R-ESIHE

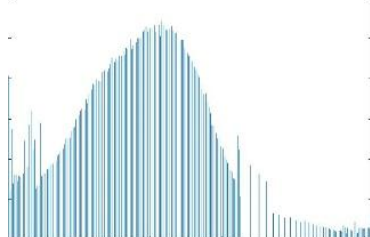

Histogram of R-ESIHE

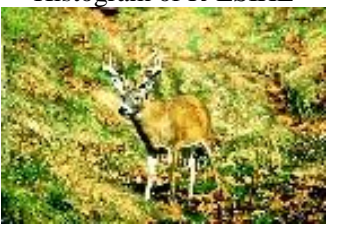

Proposed

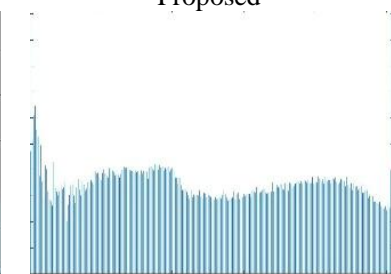

Histogram of Proposed

Fig.9. BSD Image 346016 of 'Deer' and output of different image enhancement methods 


\section{Conclusion}

In this paper, an image enhancement method is suggested where image histogram is fitted by minimizing the sum of squared residuals to reduce the actual distance of a number of pixels for each intensity value by high degree multinomial curvature fitting. Then resampling of curvature fitted data is done. After then histogram clipping threshold value is computed through central moment on the resampled data to limit the proportion of over enhancement. Then histogram is segmented equally into two section followed by equalization of sub image histogram. The results found from Matlab simulation demonstrates that the present method offers best quality of image compared to the other histogram-based and non-histogram-based contrast enhancement methods. Both the quantitative and qualitative performance assessment show the superiority of the proposed method in terms of contrast, color and brightness associated to the other compared methods.

\section{References}

[1] R. Nithyananda, A. C. Ramachandra and Preethi, "Review on Histogram Equalization based Image Enhancement Techniques," 2016 International Conference on Electrical, Electronics, and Optimization Techniques (ICEEOT), Chennai, 2016, pp. 25122517.

[2] Adaptive Image Enhancement based on Bi-Histogram Equalization with a clipping limit q Jing Rui Tang, Nor Ashidi Mat Isa

[3] CLAHE method Zuiderveld, Karel. "Contrast Limited Adaptive Histograph Equalization." Graphic Gems IV. San Diego: Academic Press Professional, 1994. 474-485.

[4] K. Singh, R. Kapoor, Image enhancement using exposure-based sub image histogram equalization, Pattern Recogn. Lett. 36 (2014) 10-14.

[5] K. Singh, R. Kapoor, S. K. Sinha, "Enhancement of low exposure images via recursive histogram equalization algorithms", Optik-Int. J. Light Electron Opt., vol. 126, no. 20, pp. 2619-2625, 2015.

[6] Singh, K., Kapoor, R.: 'Image enhancement via median-mean based sub image-clipped histogram equalization', Optik., 2014, 125, (17), pp. 4646-4651

[7] Ibrahim and N. S. Pik Kong, "Brightness Preserving Dynamic Histogram Equalization for Image Contrast Enhancement," in IEEE Transactions on Consumer Electronics, vol. 53, no. 4, pp. 1752-1758, Nov. 2007.

[8] S. C. Huang, F. C. Cheng and Y. S. Chiu, "Efficient Contrast Enhancement using Adaptive Gamma Correction with Weighting Distribution," IEEE Transactions on Image Processing, Vol. 22, No. 3, 1032-1041, March 2013.

[9] Yu W, Qian C, Baeomin Z. Image enhancement based on equal area dualistic sub-image histogram equalization method. IEEE Trans Consum Electron 1999; 45:68-75.

[10] Yeong-Taeg Kim, "Contrast Enhancement Using Brightness Preserving Bi-Histogram Equalization," IEEE Trans Consumer Electronics, vol. 43, no. 1, pp. 1-8, Feb. 1997.

[11] Soong-Der Chen, A. Rahman Ramli, "Preserving brightness in histogram equalization-based contrast enhancement techniques,” Digital Signal Processing, 12(5), pp.413-428, September 2004.

[12] Yu Wan, Qian Chen and Bao-Min Zhang., "Image Enhancement Based on Equal Area Dualistic Sub-Image Histogram Equalization Method," IEEE Trans Consumer Electronics, vol. 45, no. 1, pp. 68-75, Feb. 1999.

[13] S. Chen and A. Ramli, "Contrast enhancement using recursive mean-separate histogram equalization for scalable brightness preservation,” IEEE Trans. Consumer Electronics, vol. 49, no. 4, pp. 1301-1309, November 2003.

[14] He, Kaiming. "Single Image Haze Removal Using Dark Channel Prior." Thesis, The Chinese University of Hong Kong. 2011.

[15] Dubok, et al. "Single Image Dehazing with Image Entropy and Information Fidelity." ICIP. 2014, pp. 4037-4041.

[16] MATLAB and Statistics Toolbox Release 2018a, The MathWorks, Inc., A Natick ed., Massachusetts, United States.

[17] Arbelaez, P., Maire, M., Fowlkes, C., et al.: 'Contour Detection and Hierarchical Image Segmentation', IEEE Transactions on Pattern Analysis and Machine Intelligence., 2011, 33, (5), pp. 898-916

[18] K. Gu, D. Tao, J. Qiao and W. Lin, "Learning a No-Reference Quality Assessment Model of Enhanced Images with Big Data," in IEEE Transactions on Neural Networks and Learning 314 Systems, vol. 29, no. 4, pp. 1301-1313, April 2018. 315

[19] Sandra Lach Arlinghaus, PHB Practical Handbook of Curve Fitting. CRC Press, 1994.

[20] Numerical Methods in Engineering with MATLAB®. By Jaan Kiusalaas. Page 24.

[21] resample, [Online] Available: https://in.mathworks.com/help/signal/ref/resample.html, access date: September 2020

[22] Ed Sutton. "Histograms and the Zone System". Illustrated Photography. Archived from the original on 2015-02-23. Retrieved 2015-08-31.

\section{Authors' Profiles}

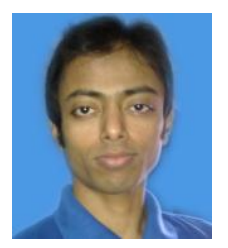

Kuldip Acharya received his M.Tech in Computer Science and Engineering (CSE) from Tripura University (a central university), Tripura, India in 2012. He is doing Ph.D in Computer Science \& Engineering from National Institute of Technology Agartala, India from 2013. His research areas of interest are image processing, computer vision, and Computer Animation. 


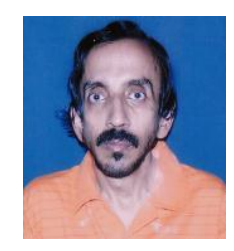

Dr. Dibyendu Ghoshal has received his B.Sc. (Honors in Physics), B.Tech \& M. Tech in Radio physics and Electronics, from Calcutta University (CU) in 1981, 1985 and 1987 respectively. He joined Indian Engineering Services (Group-A) in 1988 and served the Department of Telecommunication, GOI. He was awarded SRF in 1992 by CSIR. Subsequently, he was awarded Postdoctoral Research Associateship in 1996 and Ph.D. in Radio physics \& Electronics from CU in 1997 with a specialization in Microwave and millimeter-wave systems. His research interest includes micro \& millimeter wave, semiconductor physics \& devices, Digital Image Processing, computer vision, and computer animation.

How to cite this paper: Kuldip Acharya, Dibyendu Ghoshal, "Central Moment and Multinomial Based Sub Image Clipped Histogram Equalization for Image Enhancement", International Journal of Image, Graphics and Signal Processing(IJIGSP), Vol.13, No.1, pp. 1-12, 2021.DOI: 10.5815/ijigsp.2021.01.01 